\title{
Lossy Compression for Raw Satellite Images Using Intra Frame and Inter Frame Coding
}

\author{
Aseel M. Mahdi ${ }^{1}$, Loay E.George ${ }^{2}$, Faisal G. Mohammed $^{3}$ \\ ${ }^{1}$ Baghdad University, College of Science, Department of Computer Science, Baghdad, Iraq \\ ${ }^{2,3}$ Baghdad University, College of Science, Department of Remote Sensing \& GIS, Baghdad, Iraq
}

\begin{abstract}
The huge size of the satellite images data leads to big problems in storage capacity and power exhaustion for the transmission, therefore great efforts have been made to find low-complexity compression algorithms. To overcome these issues in this field we have proposed satellite image compression method based on inter-intra frame coding, to compress sequence of satellite images captured at different times, form different sensors, or /and different view points for the same area. Inter-frame coding principle based on removing temporal redundancy exists between two successive satellite images: a historical captured image (reference image) and a new captured image (sensed image) for the same scene. Since sequential satellite images contain almost same materials with only minor movements according to the time interval between them (several days, several months). The resulting difference's image after subtraction has much lower entropy and can be compressed with much less cost than the original sensed satellite image. This is followed by removing still existing redundancy of pixels within resulting difference's image using principle intra-frame coding, and this will be helpful in order to satisfy high compression performance while keeping the quality of the compressed satellite image as much as possible.
\end{abstract}

Keywords: Satellite images, Image Compression, Inter-Frame Coding, Intra-Frame Coding

\section{Introduction}

Data is a collection of information and redundancy, information must be kept to correctly interpret the meaning of data, while unnecessary redundancies could be removed from original data file using any of compression methods to minimize data file size [1] and facilitate dealing with large data file especially satellite images through minimizing needed storing space and maximizing loading speed. The main redundancy types are: the spatial redundancy, which representing the correlation between neighboring pixels within image, the temporal redundancy which representing the correlation between adjacent frames or between successive satellite image taken for the same area under different condition, and the spectral redundancy which representing the similar responding from pixels when some of overlapped sensors captured neighboring bands [2]. The proposed algorithm deals with spatial and temporal redundancy through implementing Inter-intra frame coding principle. Inter-frame coding responsible for removing temporal redundancy between the reference and the sensed satellite images to extract new data changed by time and other factors, this can be performed by implementing an automatic image registration method between reference and sensed satellite images to facilitates comparison between these two images, then subtract corresponding feature values in the two images to extract the new information in the sensed satellite image [3], while Intra-frame coding process responsible for removing any remaining redundancy of pixels within the image itself. Intra-frame coding stage includes implementing lossy or lossless compression methods. In our previous paper [3] we used the lossless compression method LZW as the intra-frame stage, also the paper [4] discuss the using of standard lossy compression method JPEG as the intra-frame stage, but in this paper intraframe coding stage is achieved using a hybrid compression process; this includes implementing biorthogonal (9/7) wavelet transform coding to compact energy in a few transform coefficients. Then the retained coefficients from wavelet result are quantized using scalar quantization without making any significant distortions in the image quality. After this we will obtain a large sequence of zeros which should be removed using quadtree encoding algorithm to prune these sparse blocks, finally applying shift coding algorithm to remove any still existing redundancy for the resulted image data and this finished the proposed method.

\section{Related Works}

Here are some of recent typical compression methods which applied to compress satellite images:-

- Md. Al Mamun et al. [2] implemented a lossless compression method for satellite image which based on improve the temporal correlation through using reversible integer wavelet transformation to improve the compression performance.

- Aseel et al. [3] proposed a lossless satellite images compression using Inert-frame coding principle and Lempel-Ziv-Welch (LZW) compression method. The introduced scheme attained CR (compression ratio) equal to 4.2 .

- Ahmed Hagag et al. [5] implemented a novel compression technique for satellite image based on rate control, where he divided image bands into two groups and encode each band at different bit rate, and he test the proposed technique using satellite images taken from different sensors. The proposed technique was compared with traditional satellite image compression techniques and the results show lower impact than that of traditional techniques.

- Ke -Kun H. et al. [6] proposed an improved binary tree coding with adaptive scanning order (BTCA) by optimized truncation. He used 9/7-tap biorthogonal wavelet filters for wavelet transform then divided the image into several 


\section{International Journal of Science and Research (IJSR) \\ ISSN (Online): 2319-7064}

Index Copernicus Value (2015): 78.96 | Impact Factor (2015): 6.391

blocks to be encoded individually, then he optimized truncation to obtain higher compression ratio and less memory space

- Meishan Li et al.[7] performed three-level wavelet on remote sensing images using biorthogonal wavelet,then used Set Partitioning In Hierarchical Trees (SPIHT) Algorithm which improved by fixing the threshold to compress image at fixed scale, then compare the obtained results with Embedded Zero-tree Wavelets (EZW) algorithm. The proposed algorithm outperforms EZW in terms of PSNR, compression and decompression time.

- Sendamarai.P et al. [8] introduced a compression scheme for satellite images based on using the Le Gall's 5/3 architecture with lifting. Where all the implemented operations in this architecture are done using shift operations which reduce implementation overhead. The best PSNR is achieved is $29.81 \mathrm{~dB}$.

- Xingsong Hou et al. [9] proposed a Synthetic aperture radar (SAR) complex image data compression algorithm based on quadtree coding in wavelet transform domain , where the quadtree coding (QTC) algorithm achieves the best performance for SAR complex image compression than zerotree coding algorithm.

- Shichao Zhou et al. [10] introduced a review of recently remote sensing image compression and dividing these methods into: predictive coding, transform coding, Region Of Interest (ROI)-based compression methods and taskdriven coding methods.

- V. Bhagya Raju et al.[11] submitted an improved Set Partitioning in Hierarchical Tree (SPIHT) algorithm with 2D -Discrete wavelet transforms(DWT) for multispectral image compression for various band images with high resolution

- LIU XiJia et al. [12] Implemented a novel remote-sensing image compression, based on using priori-information collection of historical remote-sensing images and registration technique to remove temporal correlation between newly-captured remote-sensing images and historical ones. The results outperform JPEG2000 and JPEG by over 1.37 times for lossless compression.

\section{Proposed Method}

The proposed compression technique has two main steps are: - Inter-frame coding stage and the Intra-frame coding which are described in the following subsections.

\subsection{Preprocessing Stage}

Usually the loaded satellite images surrounding with empty regions, which can affect on the matching operation between the two images (reference and sensed), therefore these empty regions must be cat away to ensure accurate matching operation as much as possible through checking every image line-by - line /column - by- column through all pixels in each dimension until meeting a pixel with another color, then cut away the border.

Also the captured satellite image may includes nonlinear effects caused by display system, such as : lack of dynamic range in the sensor, looks too bright or too dark, poor illumination, therefore it should be enhance using histogram equalization rule to increase the dynamic range of the image histogram and obtained a uniform distribution for pixels' intensities. Histogram equalization works to redistributing pixels' intensities to allow areas of lower contrast to obtain a higher contrast without changing the global contrast, so the histogram range will be increase [13], its formula can be written as following:

$$
O i=\left[\sum_{j=0}^{i} N\right] * \frac{\text { Max.IntensityLevel }}{\text { TotalNo.ofPixels }}
$$

Where

- Oi: the new intensity value.

- $i$ : represent the previous intensity level.

- $\left[\sum_{j=0}^{i} N\right]$ : The no. of pixels having the intensity below the output intensity level or equal to it,

- Max. Intensity Levels: the maximum intensity level which a pixel can get. For grayscale domain it equal to 255 .

\subsection{Inter-Frame Coding Stage}

To find the new added information in the sensed image, we need to match it with reference image using registration technique then we can subtract them to find the new added information (difference's image). This includes the following steps, figure (1):

a) Image features (key pints) should be detected for both images (reference, sensed).

b) The correspondence between both images must be finding.

c) The wrong matching between both images (reference, sensed) is eliminated.

d) The distorted sensed image should be corrected using affine transformation to map the points of the sensed image to the corresponding points in the reference image.

e) The transformed sensed image is subtracted from the reference image to obtain the resulting differences data image.

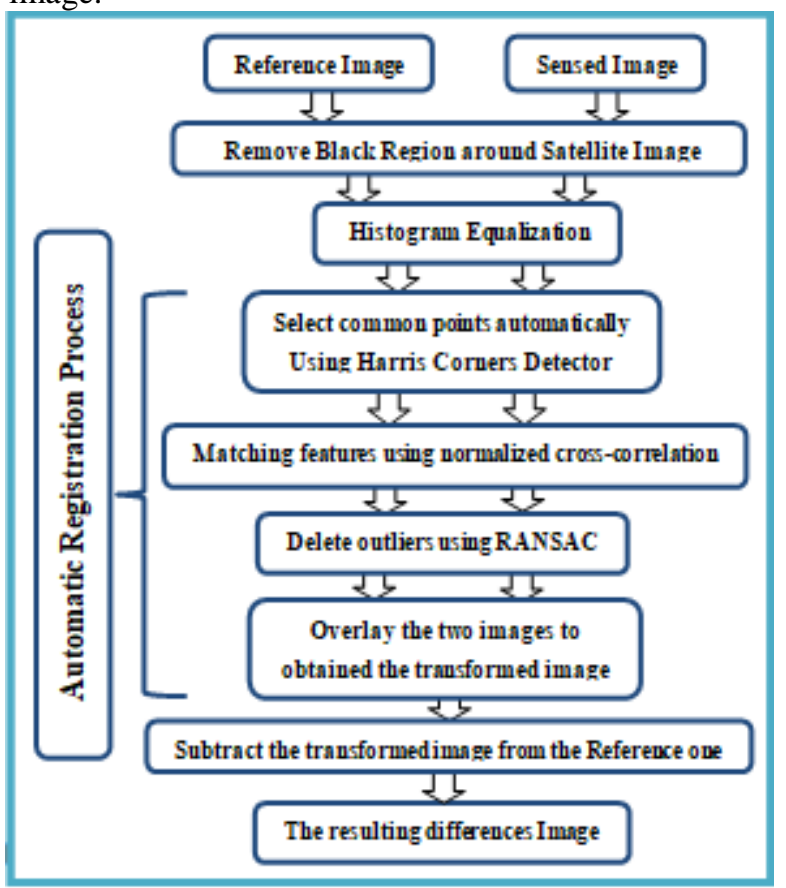

Figure 1: Inter-frame coding stage 


\section{International Journal of Science and Research (IJSR) \\ ISSN (Online): 2319-7064}

Index Copernicus Value (2015): 78.96 | Impact Factor (2015): 6.391

The following subsections explain inter-frame coding stage in details.

\subsubsection{Image Registration}

It can be define as the operation used to geometrical align two or more images captured under different conditions (different sensors, different viewpoints, and at different times) [14], the used registration process in this paper is the feature-based model, which involves using Harris corner detector to automatically detect features of type corners [3].

a) Features extracting: Harris corner detector method depends on extracting feature points of type corners. Where corner represents a good feature point and can be detected because it has a large variation for its gradient in both directions. Harris detector based on Moravec's corner detector and has the following procedure [15]:

1) Compute the two derivatives (horizontal and vertical) for both images.

2) Apply Gaussian smoothing filter to ensure that the derivative is not picking up any noisy bits (Equation 2).

3) Compute the three terms in the matrix $M$. (Equation 3).

4) Compute $C$ (Equation 4) and determine if the detected good feature is a corner or not considering the given threshold.

$$
\begin{gathered}
G(x, \sigma)=\frac{1}{2 \pi \sigma^{2} e^{-x^{2} / 2} \sigma^{2}} \\
M=\sum_{x, y} w(x, y)\left(\begin{array}{ll}
I x I x & I x I y \\
I x I y & I y I y
\end{array}\right. \\
C=|M|-k(\text { traceM })^{2}=\lambda_{1} \lambda_{2}-k(\lambda 1+\lambda 2)^{2}
\end{gathered}
$$

Where:

$\sigma$ : sigma, its value is should be $1-3,[13]$.

$w(x, y)$ :Gaussian blur weighting $\operatorname{window}(\mathrm{G})$ at position $(\mathrm{x}, \mathrm{y}, \sigma)$ is built around each candidate corner as a kind of smoothing the image prior to taking the derivative, so the derivatives will be more accurate[13].

$\left(\begin{array}{ll}I x I x & I x I y\end{array}\right)$ : Harris matrix represents products of the

I $x$ I y Iy $I y$

gradients (Ix, Iy).

$C$ : Harris point response, where $C$ is positive for corners, negative for edges, and small for flat regions [16].

$(\lambda 1, \lambda 2)$ : are the Eigen values of the matrix. If both values are greater than given threshold it will be consider a good chosen feature (corner).

$\mathrm{K}$ : tunable parameter, default value in the range of 0.04 to $0.06[16]$.

b) Feature matching: After find the features for both images (reference, sensed) using harries corner detector; we need to find matching between two feature vectors. There are different methods to find features matching such as: Euclidean distances, mutual information, normalized cross correlation [17]. In this paper the chosen method is normalized cross correlation (NCC) because it's more robust to translation, brightness and contrast changes [18]. (NCC) rule work to directly compare the intensities in small patches around each feature point.

The matching operation will result some of wrongly matching point pairs because the appearance of image features will vary from image to image in orientation, scale, and subject to affine deformations, for this reason these wrongly correlated matching point pairs should be removed using the robust estimator (RANdom SAmple Consensus) RANSAC algorithm [19]; so the resulting contains only the correct matching points that represent the actual matches between both images (sensed, reference).

RANSAC is an iterative algorithm proposed by Fischler and Bolles, used to find best partition of interest points in inlier and outlier sets and estimate a mathematical model from the inlier set [4]. In a single iteration a subset of matches is picked (random sample of 4 correspondence keypoints), and homography model $\mathrm{H}$ is then computed, then these matches are transformed using this homography and their distance to where those transforms should be computed, the matching points consider as inlier and non matching points will consider as outliers[20].

c) Apply transformation: satellite images are subject to geometric distortions due to several factors such as sensor optics, the motion of the scanning system, the velocity, and the rotation of the earth. Therefore the two images should be spatially aligned to each other and the geometry of one image must be transmitting to that of the other [21]. This can be accomplished using many models such as: Isometry transformation, similarity transformation, affine transformation, etc [20]. In this paper the applied transformation was the 1st order affine transformation which represent a combination of linear and translation transformation. This transformation has six unknown parameters two for translation, one for rotation, one for scaling, one for scaling direction, and one for scaling ratio, and can be define as [3]:

$$
\begin{aligned}
& x=m 1+m 2 X+m 3 Y \\
& y=n 1+n 2 X+n 3 Y
\end{aligned}
$$

Where: $(\mathrm{m} 1, \mathrm{~m} 2, \mathrm{~m} 3)$ and $(\mathrm{n} 1, \mathrm{n} 2, \mathrm{n} 3)$ are the transformation coefficients.

\subsubsection{Quantify differences image}

The last step in inter-frame coding stage is to apply a simple subtraction between the reference and new aligned image which will produce some negative values, therefore adding 128 to the subtraction result and applied clipping operation using the following procedure [4]:

$$
\begin{aligned}
& M \leftarrow \operatorname{image} 2(x, y) \text { - image1 }(x, y) \text {; } \\
& M \leftarrow M+128 \text {; } \\
& M i=\left\{\begin{array}{cc}
255 & \text { if } M_{i}>255 \\
0 & \text { if } M_{i}<0
\end{array}\right.
\end{aligned}
$$

Where: $\boldsymbol{M}$ represents the subtraction result (difference result).

\section{Volume 6 Issue 12, December 2017}




\subsection{Intra-Frame Coding Stage}

As mentioned earlier the aim of this stage is to eliminate the correlation for adjacent pixels within the obtained differences image by leaving a small number of significant transform coefficients that are important to the appearance of the image and discard a large number of insignificant coefficients that does not affect the visual quality of it. This stage involves 2 main steps compressor and de compressor as shown in figure (2).

The compressor unit as shown in (figure 2(A)) includes the following steps:

a) The biorthogonal (9/7) wavelet transform coding must be implemented to decompose image's signal to the known sub bands (LL, LH, HL, and HH).

b) The retained coefficients from wavelet result must be quantized using the hierarchal scalar quantization without making any significant distortions in the image quality.

c) After quantization step, we obtain a large sequence of zeros in (HL, LH, and $\mathrm{HH}$ ) parts of the image; therefore quadtree has been adopted to remove these empty regions.

d) Implementing Shift coding technique to remove the still existing statistical redundancy and obtained an efficient compression performance for the resulted data buffer and this finish the proposed compression system.

While the de-compressor unit as shown in figure 2(B) includes the following steps:

a) Applied shift decoder to reconstruct compressed components depending on short and long codewords.

b) Retrieve the empty blocks using Dequadtree.

c) Performing Dequantization process.

d) Backward biorthogonal Tap 9/7 wavelet transforms.

e) Obtained the reconstructed image.

The resulting difference image

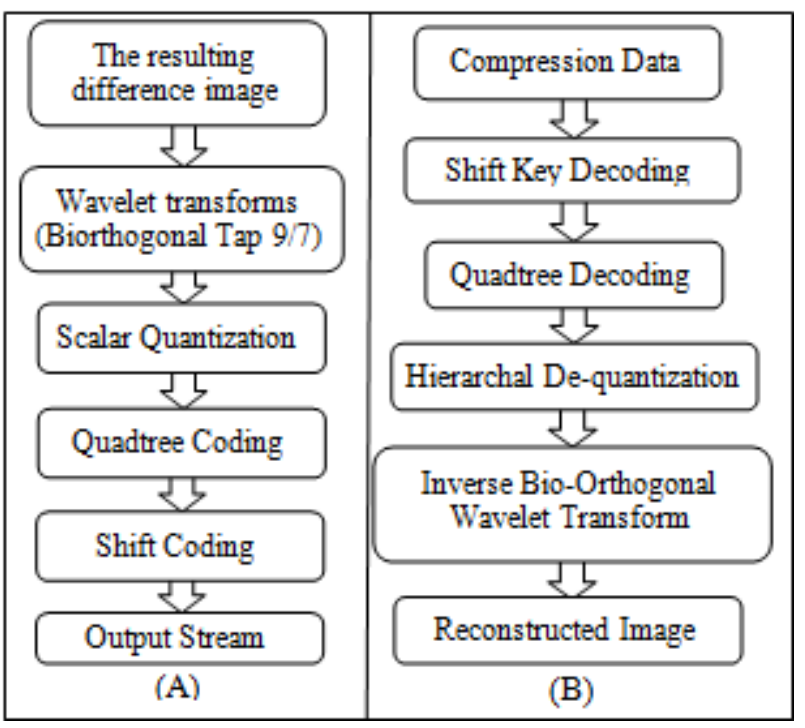

Figure2: Intra-frame coding main steps

(A): the compressor unit $\quad$ (B): the De-compressor unit

\subsubsection{Compressor unit}

The first step of compressor unit includes using wavelet filters, where the most popular wavelet filters are: Harr wavelet, Biorthogonal wavelet (Tab 3/5, Tab 9/7). The biorthogonal systems decomposition are efficient for lossy and near losses image compression, therefore they used in JPEG2000 [22]. For this reason Biorthogonal wavelet (tap 9/7) was used in this paper.

Biorthogonal wavelet: also named tap (9/7) because of the filter lengths are 9 and 7 for low and high pass filters respectively. It presents the following features:

a) The coefficients are either real numbers or integers for each filter;

b) each filters will introduced either even or odd orders;

c) Also the low pass filter is always symmetric while the high pass filter is either symmetric or antisymmetric [23].

To compute the (9/7) wavelet of an array $\mathrm{x}$ of $\mathrm{m}$ samples, where $(0 \leq \mathrm{n} \leq \mathrm{m} / 2)$ the following equations are used:

(a) Lifting step

$$
\begin{gathered}
y(n+1)=y(n+1)+a[y(n)+y(n+2)] \forall \text { odd } n \\
y(n)=y(n)+b[y(n-1)+y(n+1)] \forall \text { even } \mathrm{n} \\
y(n+1)=y(n+1)+c[y(n)+y(n+2)] \forall \text { odd } \mathrm{n} \\
y(n)=y(n)+d[y(n-1)+y(n+1)] \forall \text { even } \mathrm{n} \text { in the }
\end{gathered}
$$
range

(b) Scaling step, $\mathrm{k}$ is the scaling factor

$$
\begin{gathered}
y(n+1)=k^{*} y(n+1) \forall \text { odd } \mathrm{n} \text { in the range } \\
y(n)=\frac{y(n)}{k} \forall \text { even } \mathrm{n} \text { in the range }
\end{gathered}
$$

By reversing the transform operation this can be used to obtain the inverse wavelet transformation, the values of the five coefficients are listed in table (1).

Table 1: biorthogonal Tap 9/7

Wavelet filter coefficients [24]

\begin{tabular}{|c|c|}
\hline Coefficient & Value \\
\hline $\mathrm{a}$ & -1.586134342 \\
\hline $\mathrm{b}$ & -0.05298011854 \\
\hline $\mathrm{c}$ & 0.8829110762 \\
\hline $\mathrm{d}$ & 0.4435068522 \\
\hline $\mathrm{k}$ & 1.230174105 \\
\hline
\end{tabular}

Quantization module: To reduce the number of bits required to store the transformed coefficients from wavelet result, quantization should be implemented, it represent the heart of any lossy compression scheme which assure the low frequency data, and try to moving the high frequency data towards zero, because the zero doesn't need memory[25].

The following equation has been applied to quantize the coefficients of each wavelet subband (HL, LH, and $\mathrm{HH}$ ) individually with an appropriate $Q_{\text {step }}$ using the following equation [26]:

\section{Volume 6 Issue 12, December 2017}




\section{International Journal of Science and Research (IJSR) \\ ISSN (Online): 2319-7064}

Index Copernicus Value (2015): 78.96 | Impact Factor (2015): 6.391

$$
\begin{aligned}
& Q_{\text {step }}(n)= \\
& \left\{\begin{array}{cl}
Q \alpha^{\text {Npass-1 }} & \text { for LH and HL sub bands } \\
Q \beta \alpha^{\text {Npass-1 }} & \text { for HH sub bands where } Q \geq 1(14)
\end{array}\right.
\end{aligned}
$$

Where:

$\mathrm{N}$ : symbolize wavelet level number (i.e., the pass number), $\alpha$ : represent the rate of increase of quantization step, $\alpha \leq 1$.

$\beta$ : Additional ratio for the quantization of the $\mathrm{HH}$ subband, $\beta \geq 1$.

And the quantization indexes for every approximate (LL) and wavelet coefficients are determined using [24]:

$$
W_{Q}(x, y)=\operatorname{round}\left(\frac{W_{c}(x, y)}{Q_{S}}\right)
$$

Where, $\mathrm{W}_{\mathrm{C}}()$ is the array of the wavelet transform coefficients, $\mathrm{W}_{\mathrm{Q}}($ ) its quantization index array.

Quadtree process: The next step is to implement the recursive Quadtree algorithm to eliminate the empty blocks by dividing the input band into non-overlapped four equal regions (quadrants), if the tested quadrant contains non-zero coefficient, then the search is repeated upon its sub blocks and stop when reaching size $(2 \times 2)$ quadrant. If the quadrant was empty then it will be removed [27]. The output will be two arrays, Seq ( ): including the nonempty coefficients and Map ( ): store the indicators for quadtree mapping values (these indicators necessary for retrieving the image information).

Shift coding process: this lossless compression technique aims to remove the statistical redundancy without time consuming [28], through encoding set of values using codewords which is less than the length of the needed bits to represent these values. This process consists of the following steps as shown below in figure 3 .

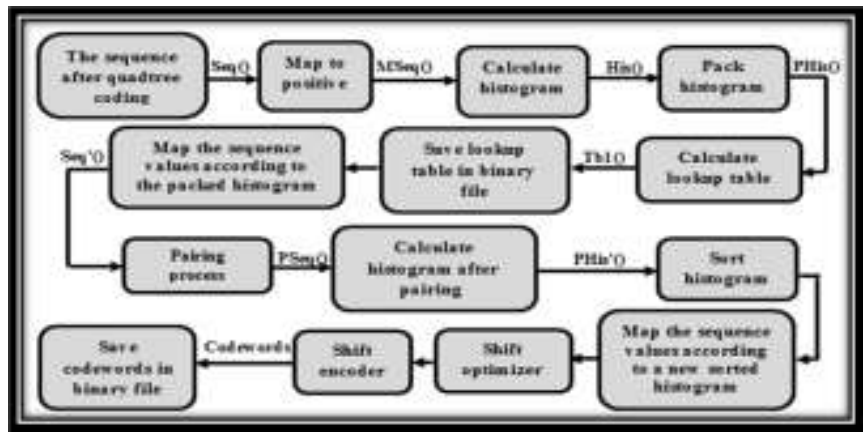

Figure3: steps of shift coding process [28]

A.Mapping to positive : It means convert the incoming sequence of values to positive numbers to avoid coding complexity, where every positive value will be representing as even number and every negative value in the incoming sequence will be representing as odd value using the following equation [27]:

$$
k(i)=\left\{\begin{array}{cc}
2 k(i) & \text { if } k(i) \geq 0 \\
-2 k(i)+1 & \text { if } k(i)<0
\end{array}\right.
$$

B.Histogram Packing: after mapping to positive, many values will not appear in the sequence which leads to produce many gaps in the histogram of that sequence. To overcome this problem the histogram should be packed to include the non-zero frequency elements only. Then these positive elements must be remapped in the histogram according to the packed elements [28].

C.Remove pairing: to achieve further compression gain, the most repeated pair of successive values should replaced by a single coefficient value such as Max +1 , where Max represent the highest value off all elements, and Max value will be increment by 1 after each pairing iteration [27].

\section{D.Histogram sorting:}

The resulted histogram after pairing process, saved as overhead information for decoding operation, and according to the indexes of the histogram's elements values, these value are remapped [28].

\section{E.Shift Optimizer}

The shift optimizer algorithm has been used to determine the two codewords lengths in order to encode the small codewords and large codewords values of the input sequence components. This optimizer search for the best short codeword length $\left(n_{s}\right)$ and the corresponding long codeword $\left(n_{l}\right)$ uses the following equations to calculate the entire number of consumed bits to encode the input symbols [27]:

$$
T_{\text {Bits }}=n_{s} \sum_{i=0}^{i=2^{S}-1} \operatorname{His}(i)+n_{l} \sum_{i=2^{S}-1}^{L} \operatorname{His}(i)
$$

Where:

$n_{s}$ : The length of short codeword, which leads to minimum possible value for $T_{\text {Bits }}$

$n_{\text {l: }}$ The length of long codeword, which leads to minimum possible value for $T_{\text {Bits }}$.

s: number of bits for short codewords.

L: number of bits for long codewords.

His (): denotes the histogram - array.

\section{F. Shift Encoder}

At this step the small coded element is coded using " 0 " value and $n_{s}$ value, while the large element is coded using " 1 " value with $n_{l}$ value.

The sum of the probability of occurrence of short codewords should be greater than the sum of the probability of long codewords. Short codewords are assigned to symbols have higher probability to reduce data size.

\subsubsection{Decompressor unit}

This unit includes implementing shift decoder to gets the actual quantized values and stored as a one dimensional array, then dequadtree is implemented to retrieve all the empty blocks and configure the values of the image as a twodimensional array. Then Dequantization technique and backward biorthogonal Tap 9/7 wavelet are implemented to obtain the reconstructed image.

\section{Results and Discussion}

Many tests are applied to the proposed scheme in terms of compression ratio (CR), peak signal to noise ratio(PSNR), mean square error (MSE)and mean absolute error (MAE) using C\# application program to evaluate the proposed

Volume 6 Issue 12, December 2017 


\section{International Journal of Science and Research (IJSR) \\ ISSN (Online): 2319-7064}

Index Copernicus Value (2015): 78.96 | Impact Factor (2015): 6.391

methodology. The used data set was the same data set used in [4]; the image given in Figure 3 was subset of tested images, Landsat7, gray scale images, each of which has 7 bands, with dimensions 818x 718 taken over Iraq/Baghdad.

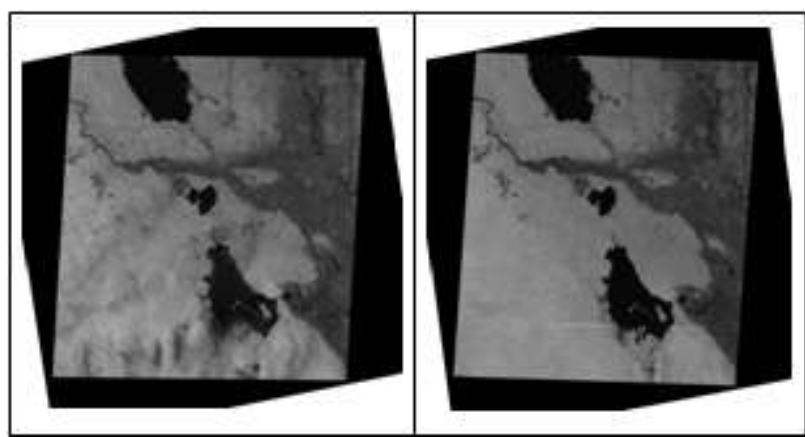

Figure 4: Image pair, band7, Path: 169, Row: 037 Left side: reference Image, taken at 12/2/2000, size :( 824X724) pixel

Right side: Sensed Image, taken at 31/3/2000, size: (823X724) pixel

The preprocessing step is includes removing the black area surrounding the obtained satellite image and enhance satellite image contrast. This step is very necessary to increase the accuracy of registration operation; figures $(5,6)$ show the effect of using this process on Image band 1 .

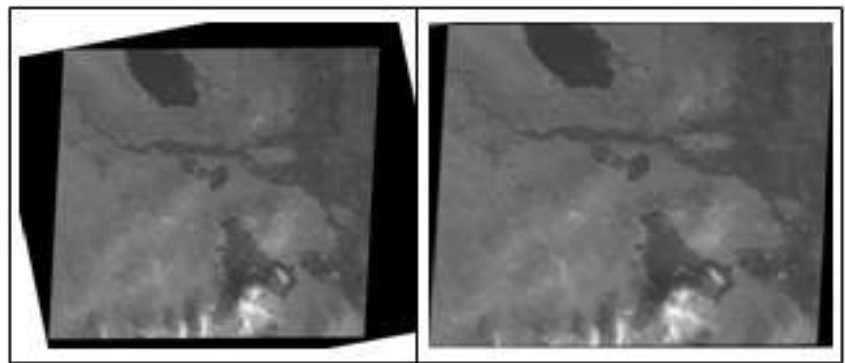

Figure 5: removing black area surrounding image band 1 Left side the original image, right side the processed image

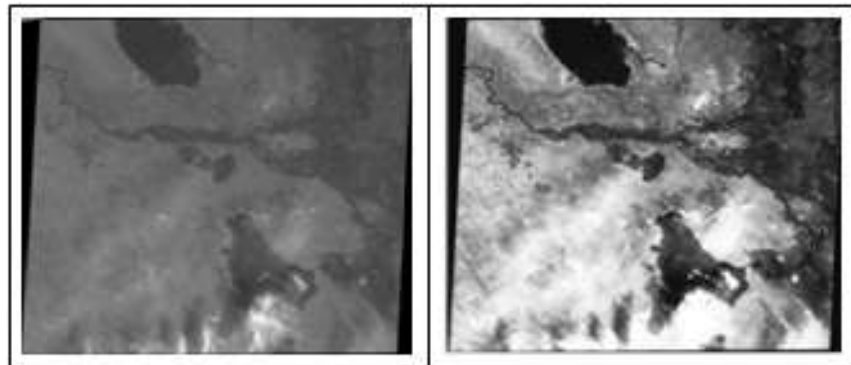

Figure 6: apply histogram equalization on Image band1

Left side the original image, right side the processed image

To evaluate the transformation operation, the measures (Mean Square Error MSE, Mean Absolute Error MAE) are computed according to the following formula [4]:

$$
\begin{aligned}
& M S E=\frac{1}{N} \sum_{y} \sum_{x}\left(\operatorname{Im} g_{1}(x, y)-\operatorname{Im} g_{2}(x, y)\right)^{2} \\
& M A E=\frac{1}{N} \sum_{y} \sum_{x}\left|\operatorname{Im} g_{1}(x, y)-\operatorname{Im} g_{2}(x, y)\right|
\end{aligned}
$$

Where, $\mathrm{N}$ is (width $\mathrm{x}$ height).
These two errors metric are computed twice, firstly: between the reference and sensed images denoted by MSE1, MAE1 and secondly: computed between the reference and the transformed Images denoted by MSE2, MAE2 as illustrated in table2, table3. The results show that the registration operation minimizes the dissimilarity between the reference image and sensed image, also improved the correlation between the two images. Figure 7 shows the obtained difference image from the inter-frame coding stage for image band3.

Table 2: using MSE metric to evaluate of transformation task

\begin{tabular}{|c|c|c|}
\hline $\begin{array}{c}\text { Image } \\
\text { Band }\end{array}$ & $\begin{array}{c}\text { (MSE1) Between Base } \\
\text { and Sensed Image }\end{array}$ & $\begin{array}{c}\text { (MSE2) Between Base and } \\
\text { Transformed Image }\end{array}$ \\
\hline 1 & 1286.18 & 691.160 \\
\hline 2 & 1046.33 & 492.059 \\
\hline 3 & 808.296 & 349.758 \\
\hline
\end{tabular}

Table 3: using MAE metric to evaluate of transformation

\begin{tabular}{|c|c|c|}
\multicolumn{3}{|c}{ task } \\
$\begin{array}{c}\text { Image } \\
\text { Band }\end{array}$ & $\begin{array}{c}\text { (MAE1) Between Base } \\
\text { and Sensed Image }\end{array}$ & $\begin{array}{c}\text { (MAE2) Between Base and } \\
\text { Transformed Image }\end{array}$ \\
\hline 1 & 15.203 & 10.950 \\
\hline 2 & 13.190 & 8.789 \\
\hline 3 & 11.233 & 7.050 \\
\hline
\end{tabular}

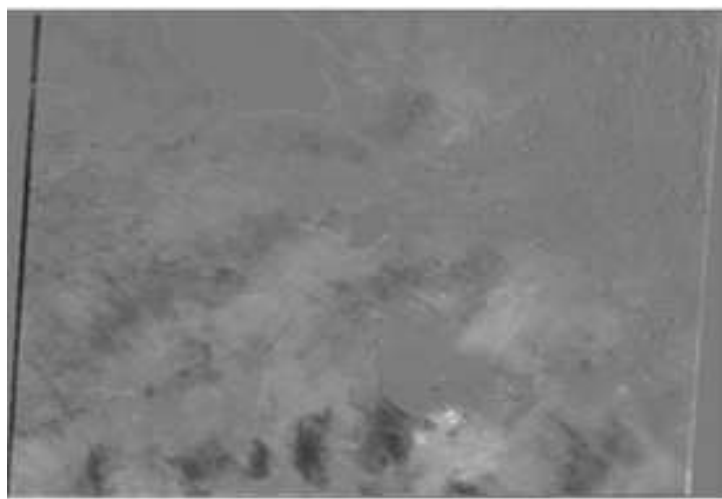

Figure7: The resulted difference images for image band3

Another measure was used; it is called Entropy measure [4], where the entropy will be high if the data distribution in the image is relatively flat and the range of values is high, while the low entropy refers to slim data distribution, its formula [3]:

$$
\begin{gathered}
E=-\sum P(i) \log P(i) \\
\text { Where } P(i)=\frac{\text { Histogram }(i)}{\text { no.ofimagePixels }}
\end{gathered}
$$

Table 4: Entropy Comparison

\begin{tabular}{|c|c|c|}
\hline Image Band & $\begin{array}{c}\text { Entropy for } \\
\text { Transformed Image }\end{array}$ & $\begin{array}{c}\text { Entropy for } \\
\text { Residue Image }\end{array}$ \\
\hline 1 & 6.889 & 5.366 \\
\hline 2 & 7.075 & 5.471 \\
\hline 3 & 7.394 & 5.278 \\
\hline
\end{tabular}

The entropy is given for both transformed and difference image for each test image band as shown above in table 4 . Which depict the significant improvement in entropy value;

\section{Volume 6 Issue 12, December 2017}




\section{International Journal of Science and Research (IJSR) \\ ISSN (Online): 2319-7064}

Index Copernicus Value (2015): 78.96 | Impact Factor (2015): 6.391

where the entropy of resulting difference image always has lower value and this indicates to that it is more appropriate for any compression scheme.

The performance of intra-frame coding system when applying shift coding process is measuring for two cases (for the compression of the entire transformed sensed image and for the compression of the resulting difference image).the using measures for compression performance: compression ratio $\mathrm{CR}$, MSE, Peak signal to noise ratio PSNR, Compression gain(CG) and time encoding/decoding. As shown in table (5). The listed results demonstrate that the compression of the resulting difference image outperforms the compression of the entire transformed sensed image by obtaining a highest compression ratio while keeping image quality, the best obtained CR when compress the resulting difference for image band 3 .

Table 5: The shift coding compression results when compress only difference image and the corresponding results generated by applying compression of the entire transformed sensed image

\begin{tabular}{|c|c|c|c|c|c|c|c|}
\hline \multicolumn{2}{|c|}{$\begin{array}{c}\text { Image band } \\
\text { number }\end{array}$} & CR & MSE & PSNR & CG & \multicolumn{2}{|c|}{ Time } \\
\cline { 6 - 8 } & encode & Decode \\
\hline \multirow{2}{*}{1} & $\begin{array}{c}\text { Sensed } \\
\text { image }\end{array}$ & 22.687 & 68.944 & 29.745 & 0.907 & 12417 & 749 \\
\cline { 5 - 8 } $\begin{array}{c}\text { Difference } \\
\text { image }\end{array}$ & 61.328 & 26.675 & 33.869 & 0.969 & 1744 & 735 \\
\hline \multirow{2}{*}{$2 \begin{array}{c}\text { Sensed } \\
\text { image }\end{array}$} & 27.447 & 58.311 & 30.473 & 0.924 & 7771 & 732 \\
\hline $\begin{array}{c}\text { Difference } \\
\text { image }\end{array}$ & 74.761 & 23.172 & 34.481 & 0.974 & 1316 & 720 \\
\hline \multirow{2}{*}{3} & $\begin{array}{c}\text { Sensed } \\
\text { image }\end{array}$ & 32.438 & 46.052 & 31.498 & 0.942 & 4700 & 733 \\
\cline { 2 - 7 } & $\begin{array}{c}\text { Difference } \\
\text { image }\end{array}$ & 102.614 & 16.327 & 36.001 & 0.980 & 994 & 718 \\
\hline
\end{tabular}

The above results show that:

1) A highest gain in (PSNR, CR) when applying the proposed scheme to compress only the resulting difference image, where the best obtained CR is 102.614 for Image band3 with PSNR equal to 36.001.

2) Lowest (MSE) when applying the proposed scheme to compress only the resulting difference image which means preserving image quality.

3) A highest gain in encoding and decoding time when applying compression scheme to compress only the resulting difference image.

\section{Conclusion}

This paper introduced a lossy compression scheme for satellite images that benefit from the presence of historical archives for the satellite images to compress new satellite images more effectively, through applying inter-frame coding and intra -frame coding techniques which applied compression on the new added information only in the sensed satellite image instead of compress the whole entire transformed sensed image, thus satisfy more efficient compression. The numerical simulation results show that the proposed scheme outperforms compression of the entire incoming sensed satellite image, where the best obtained CR is (102.614).

\section{References}

[1] Salomon Dav., Data Compression the Complete Reference, Third Edition, Springer-Verlag London, 2007.

[2] Al Mamun Md., Ali Hossain Md., Nazrul Islam Mondal and Aktar Mumu, "Satellite image compression using integer wavelet regression", International Conference on Electrical Computer and Communication Engineering (ECCE). Cox's Bazar. Bangladesh, February 2017.

[3] Mahdi Aseel M. and Mohammed Faisal G., "lossless compression scheme for satellite images using Interframe coding with LZW coding", Australian journal of basic and applied science, ISSN:1991-8178 EISSN: 2309-8414. Pages: 86-95, Vol.11, No.9, June 2017.

[4] Mahdi Aseel M., George Loay E., Mohammed Faisal G., "The Effect of Using Inter-Frame Coding with Jpeg to Improve the Compression of Satellite Images", Journal of Science, Vol. 58, No.4A pp: 1970-1978, DOI: 10.24996/ ijs.2017.58.4A.19, 2017.

[5] Ahmed Hagag, Xiaopeng Fan, Fathi E. Abd El-Samie, "Lossy compression of satellite images with low impact on vegetation features", Multidim Syst Sign Process DOI 10.1007/s11045-016-0443-y.@ Springer Science+ Business Media New York, 2016.

[6] Ke-Kun Huang,Hui Liu,Chuan-Xian Ren,Yu-Feng Yu,Zhao-Rong Lai, "Remote sensing image compression based on binary tree and optimized truncation”, Digital Signal Processing ,(C 2017 Elsevier, volume 64, Pages 96-106, May2017.

[7] Meishan Li, Lihua Sheng, Yanchun Ming, "Compression of Remote Sensing Images Using an Improved SPIHT Algorithm", 3rd Asian Pacific Conference on Energy, Environment and Sustainable Development (APEESD 2017) ISBN: 978-1-60595-4356, 2017.

[8] Sendamarai.P, M.N.Giri Prasad, Madhava Prabhu S, and Nagesh K.N, "A CDF based Lifting scheme for the satellite image compression". 3rd MEC, International Conference on Big Data and Smart City, 2016.

[9] X.S. Hou, M. Han, C. Gong, X.M. Qian, "SAR complex image data compression based on quadtree and zerotree coding in discrete wavelet transform domain: a comparative study", Neurocomputing 148 561-568, 2015.

[10] Shichao Zhou, Chenwei Deng, and Baojun Zhao, "Remote sensing image compression: R review". IEEE, International Conference on Multimedia Big Data, 2015.

[11] V.B. Raju, K.J. Sankar, C.D. Naidu, S. Bachu, Multispectral image compression for various band images with high resolution improved DWT SPIHT, Int. J. Signal Process., Image Process. Pattern Recognit. 9 (2) 271-286, 2016.

[12] XiJia LIU, XiaoMing TAO and Ning GE, "Remote sensing image compression using priori information and feature registration", Vehicular Technology Conference (VTC Fall), 978-1-4799-8091-8/15, IEEE 82 ${ }^{\text {nd }}, 2015$.

[13] Frank Y.shih," Image processing and pattern recognition Fundamentals and Techniques, Copyright 2010 by the Institute of Electrical and Electronics Engineers, Inc ISBN 978-0-470-40461-4 (cloth), 2010.

[14] Jimmy S., "Technique of Image Registration in Digital Image Processing-A review", International Journal of 


\section{International Journal of Science and Research (IJSR) \\ ISSN (Online): 2319-7064 \\ Index Copernicus Value (2015): 78.96 | Impact Factor (2015): 6.391}

Information Technology and Knowledge Management, Volume 5, No. 2, pp. 239-243, July-December 2012.

[15] Edward R. and Tom Drummond, "Machine Learning for High-Speed Corner Detection”. (C) Springer Verlag Berlin Heidelberg, 2006.

[16] Biswajit Biswas, Amlan Chakrabarti, Kashi Nath Dey., "Image Registration Method using Harris Corner and Modified Hausdorff Distance with Near Set". IEEE 2nd International Conference on Recent Trends in Information Systems (ReTIS), 2015.

[17]Richard Szeliski., Computer Vision: Algorithms and Applications". Springer. ISBN-10: 1848829345 , September 30, 2010.

[18] Richard Szeliski., "Image Alignment and Stitching: A Tutorial". Handbook of Mathematical Models in Computer Vision, Springer, pages 273-292, 2006.

[19] Yumei Z., Zhen Sun and Junbao Li, Yu Peng, "An Airborne Remote Sensing Image Mosaic Algorithm Based on Feature Points", Sixth International Conference on Instrumentation \& Measurement, Computer, Communication and Control, 2016.

[20] Mohammed T., Abd El Rahman Shabayek, Hamed N., Satellite image matching and registration: A comparative study using invariant local features.. Awad A. and Hassaballah M., Image Feature Detectors and Descriptors: foundations and applications, (C) Springer international publishing Switzerland, studies in computational intelligences 630, DOI 10.1007/978-3319-28854-3, 2016.

[21] Ardeshir Goshtasby, "Image Registration: Principles, Tools and Methods (Advances in Computer Vision and Pattern Recognition)", Springer, 2012th Edition, Kindle Edition, (January 11, 2012).

[22]Loay E. George, Bushra A. Sultan.," Image Compression Based On Wavelet, Polynomial and Quadtree”. Journal of Applied Computer Science \& Mathematics, no. 11 (5), Suceava, 2011.

[23]Loay A. George, Bushra Q. Al-Abudi, and Faisel G. Mohammed., "Image Compression Based on Biorthogonal Wavelet Transforms". Journal of AlNahrain University Vol.10 (2), December, pp.178-186, 2007.

[24] Kotteri K. A., Optimal, Multiplier less Implementations of the Discrete Wavelet Transform for Image Compression Applications, M.Sc. thesis, Electrical Engineering, Blacksburg, Virginia, Apr, 2004.

[25] Ahmed, B. K.; "DCT Image Compression by RunLength and Shift Coding Techniques", Journal of University of Anbar for Pure Science, Vol. 5, No. 3, pp. 39-44, 2011.

[26] Drweesh, Z. T.; Compressive Audio Blakley Secret Sharing Using Transform Coding, M.Sc. Thesis, College of Science, Baghdad University, Iraq, 2014.

[27] Ali H. Ahmed, Loay E. George, "Color Image Compression Based on Wavelet, Differential Pulse Code Modulation and Quadtree Coding", Research Journal of Applied Sciences, Engineering and Technology 14(2): 73-79, 2017.

[28] Shaymaa D. Ahmed,"the use of cubic Bezier interpolation, biorthogonal wavelet and quadtree coding to compress color images", British journal of applied science \& technology, ISSN: 2231-0843, Vol.: 11, Issue: 4, 2015.

Volume 6 Issue 12, December 2017 